\title{
An Improved Design of Dipole Antenna Array for Base Station Applications
}

\author{
He Wang ${ }^{1, a}$, Hu Yang ${ }^{1, b}$, Lei Gu ${ }^{2, c}$ and Fei Zhao ${ }^{2, d}$ \\ ${ }^{1}$ National University of Defense Technology, Changsha, China \\ ${ }^{2}$ Southwest Electronics and Telecommunication Technology Research Institute, Chengdu, China \\ aiamkennan@163.com, byanghu90@163.com, 'gulei100121@sina.com, ${ }^{\mathrm{d}}$ bitzhaofei@163.com
}

\begin{abstract}
Keywords: dipole, broadband, wide beamwidth, stable high gain, base station antenna.
Abstract. To meet the high requirements of base station antennas, such as broadband, wide beamwidth and high gain, an improved dipole antenna array is proposed. Generally, the working frequency range of the conventional unidirectional dipole element cannot cover the $2 \mathrm{G}, 3 \mathrm{G}$ and $4 \mathrm{G}$ band $(1.71-2.69 \mathrm{GHz})$ at the same time. To solve this problem, two parasitic dipoles are added on both sides of the conventional dipole. The simulated results show that the return loss of the improved element is less than $-10 \mathrm{~dB}$ from $1.62 \mathrm{GHz}$ to $2.85 \mathrm{GHz}$, which has a complete coverage of $2 \mathrm{G}, 3 \mathrm{G}$ and $4 \mathrm{G}$ band. And its beamwidth is more than $120^{\circ}$ in the whole band. Apart from it, the gain of the element is levelling out at around $6.9 \mathrm{dBi}$. To enhance the gain further, a $1 \times 4$ antenna array is designed and it can obtain a gain of more than $11.7 \mathrm{dBi}$. Therefore, the proposed array is more profitable for the wireless mobile communication systems.
\end{abstract}

\section{Introduction}

Due to tremendous developments of various generations of wireless mobile communication systems, base station antennas have achieved dozens of milestones for a series of standards [1], such as $2 \mathrm{G}$ base station antennas for GSM and CDMA system [2], 3G base station antennas for TD-SCDMA, WCDMA, CDMA2000 [3], and 4G base station antennas for LTE/ LTE-Advanced [4].

As a mounting number of users are involved in wireless networks, an ever increasing capacity and more base station antennas are in urgent demand. In order to optimize the resource utilization efficiency, base station antenna having functional characteristics such as broad impedance bandwidth and stable gain are highly desired.

To satisfy such functional characteristics, a magneto-electric dipole antenna was proposed in [5]. This antenna had a bandwidth of $40 \%(1.82-2.73 \mathrm{GHz})$. A broadband dual-polarized planar antenna, composed of two perpendicularly crossed bowtie dipoles, is proposed in [6]. This antenna has a bandwidth of $45 \%(1.7-2.7 \mathrm{GHz})$ and a half power beamwidth (HPBW) of around $65^{\circ}$. In [7], a broadband asymmetric folded dipole antenna with high front-to-back ratio for LTE base stations is designed. The impedance bandwidth is $58.2 \%(1.62-2.95 \mathrm{GHz})$ and the HPBW is greater than $65^{\circ}$ at both principal planes in the frequency range from 1.7 to $2.7 \mathrm{GHz}$. In [8], a $\pm 45^{\circ}$ linear-polarized cross-dipole with an operating band from $1.7 \mathrm{GHz}$ to $2.7 \mathrm{GHz}$ is designed. And the HPBW is controlled by encircling the antenna with a conducting wall and placing cross-directors above the antenna, respectively, and a steady HPBW of $67^{\circ} \pm 3^{\circ}$ is obtained.

However, in many cases, wide beamwidth in the main azimuth plane and narrow ones in the elevation plane are required for base station antennas, so that the efficiency and coverage quality of the antenna can be enhanced [9].

Under this circumstance, a dipole element with a beamwidth of more than $120^{\circ}$ is proposed in this paper. Subsequently, the element is improved to obtain a broader impedance bandwidth, and the frequency range $\left(\left|S_{11}\right|<-10 \mathrm{~dB}\right)$ of the improved antenna element covers the $2 \mathrm{G}, 3 \mathrm{G}$ and $4 \mathrm{G}$ band. At last, a $1 \times 4$ array is designed to achieve a higher gain of over $12.1 \mathrm{dBi}$, which can meet the demand for broadband, wide beamwidth and high gain in the wireless mobile communication systems. 


\section{The Design of Dipole Element}

The geometry of the conventional unidirectional dipole element is shown in Fig. 1.

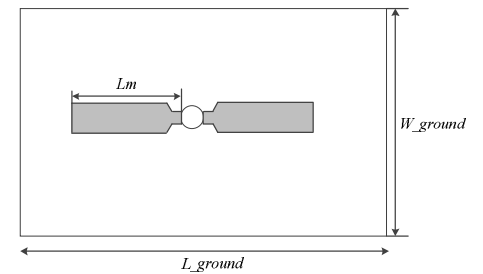

(a) Top view

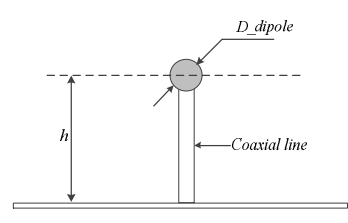

(b) Side view

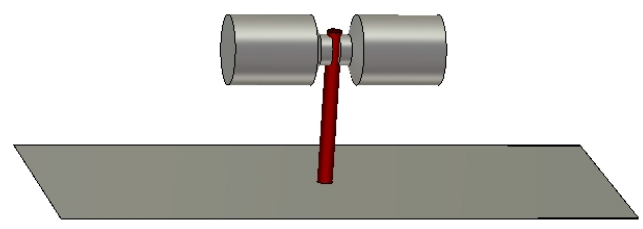

(c) 3D view

Fig. 1 Geometry of the conventional unidirectional dipole element

In order to realize the unidirectional radiation characteristic, the dipole is placed above the metallic ground plane with height $h$, which is about a quarter of the wavelength of the center frequency $(2.2 \mathrm{GHz})$. For base station applications, the optimized parameters of this element are displayed in Table 1.

Table 1 The optimized parameters of the conventional unidirectional dipole element

\begin{tabular}{|c|c|c|c|c|c|}
\hline Parameter & Lm & D_dipole & L_ground & W_ground & $h$ \\
\hline Value $(\mathbf{m m})$ & 26.5 & 18 & 150 & 65 & 35 \\
\hline
\end{tabular}

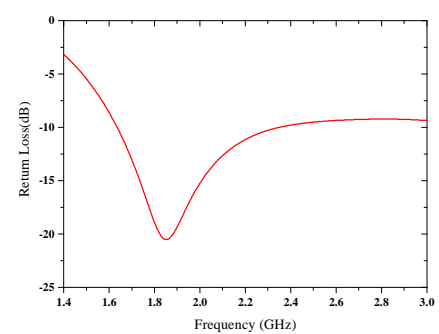

Fig. 2 Simulated return loss of the conventional unidirectional dipole element

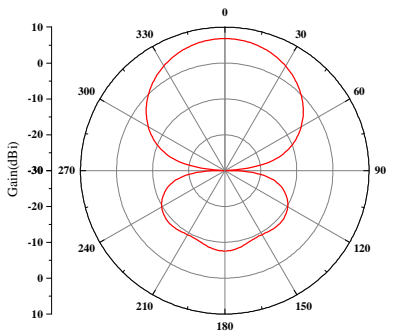

(a) E-plane

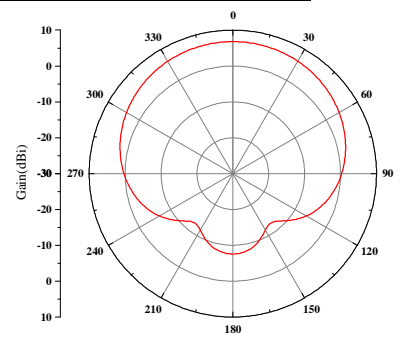

(b) H-plane

Fig. 3 Simulated radiation pattern at $2.2 \mathrm{GHz}$

Fig. 2 shows the return loss of the conventional unidirectional dipole. The return loss is less than $-10 \mathrm{~dB}$ from $1.63 \mathrm{GHz}$ to $2.35 \mathrm{GHz}$ and its impedance bandwidth is $36.18 \%$. Besides, it is obvious that the impedance bandwidth can be widened by enlarging the diameter of the dipole, but the conventional unidirectional dipole cannot fully cover the $2 \mathrm{G}, 3 \mathrm{G}$ and $4 \mathrm{G}$ band.

The simulated radiation pattern at the center frequency $2.2 \mathrm{GHz}$ is shown in Fig. 3. As can be seen, the beamwidth of H-plane and E-plane are $127.8^{\circ}$ and $58.5^{\circ}$ respectively. Compared to the base station antennas whose H-plane beamwidth is about $65^{\circ}$, an omnidirectional pattern in the horizontal plane can be realized by using 3 antennas instead of 6 or more, which is beneficial to reduce the cost of construction on a large scale. Moreover, the maximum gain of this element is $6.79 \mathrm{dBi}$, which happens at $2.2 \mathrm{GHz}$.

\section{The Design of Improved Antenna}

\section{A. An Improved Dipole Element}

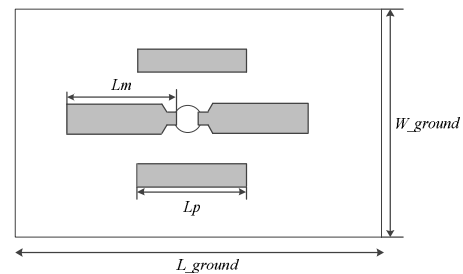

(a) Top view

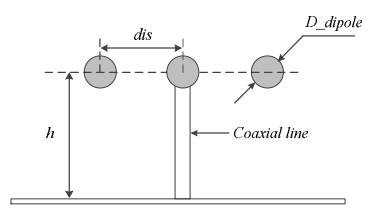

(b) Side view

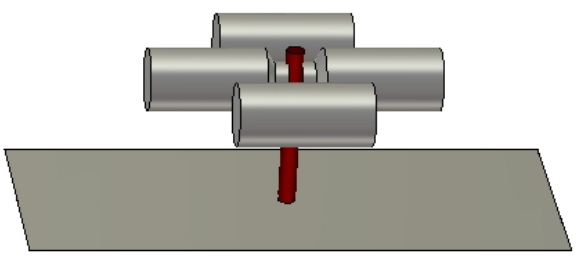

(c) $3 \mathrm{D}$ view

Fig. 4 Geometry of the improved dipole element

Generally, to reduce the complexity of the mobile communication system, base station antennas need to own the feature of broadband, but the bandwidth of the conventional unidirectional dipole element proposed in part I cannot meet this requirement. Through the study of the geometry of this 
element, it is found that a broader band can be realized by adding two parasitic dipoles on both sides of the conventional dipole. Therefore, an improved dipole element is put forward, and its structure is shown in Fig. 4. Apart from the length of parasitic dipoles $L p$ is $30 \mathrm{~mm}$, the other parameters are identical to Table 1.

Fig. 5 displays the return loss of the improved dipole element. As can be seen, the return loss is less than $-10 \mathrm{~dB}$ from $1.62 \mathrm{GHz}$ to $2.85 \mathrm{GHz}$, which fully covers the $2 \mathrm{G}, 3 \mathrm{G}$ and $4 \mathrm{G}$ band. And its impedance bandwidth is $55.03 \%$, which is $18.85 \%$ wider than the conventional dipole element.

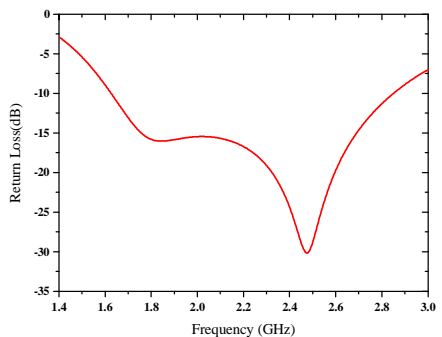

Fig. 5 Simulated return loss of the improved dipole element

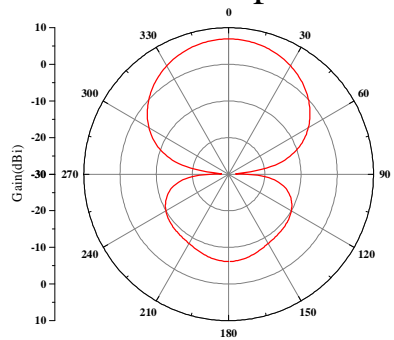

(a) E-plane

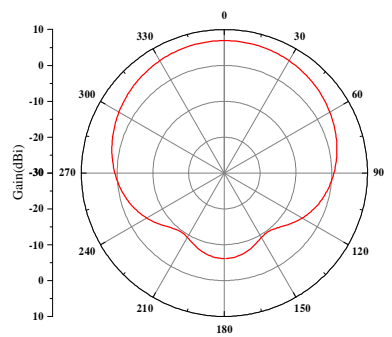

(b) H-plane

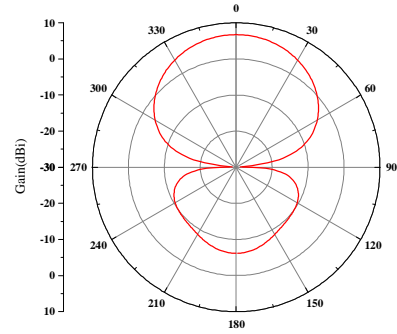

(a) E-plane

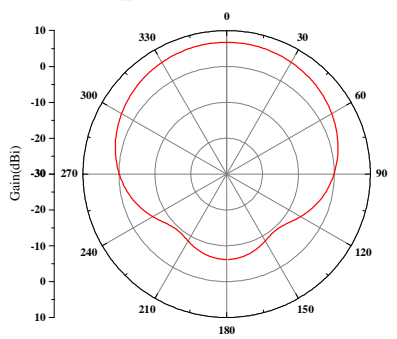

(b) H-plane

Fig. 6 Simulated radiation pattern at $1.7 \mathrm{GHz}$

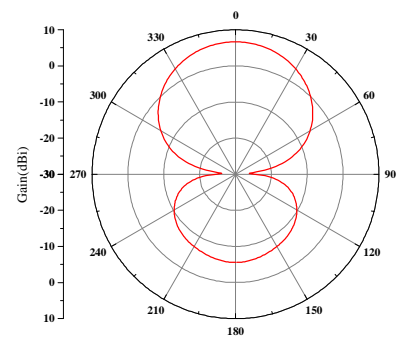

(a) E-plane

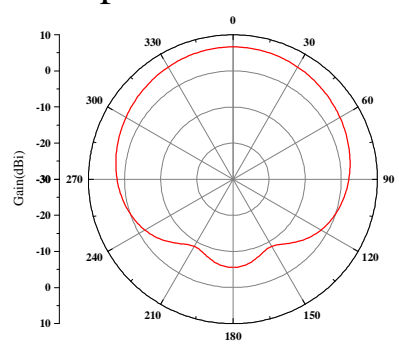

(b) H-plane

Fig. 7 Simulated radiation pattern at $2.2 \mathrm{GHz} \quad$ Fig.8 Simulated radiation pattern at $2.7 \mathrm{GHz}$

Fig. 6-Fig. 8 illustrate the radiation pattern of the improved dipole element at $1.7 \mathrm{GHz}, 2.2 \mathrm{GHz}$ and $2.7 \mathrm{GHz}$, respectively. As can be seen, the beamwidth of $\mathrm{H}$-plane is more than $120^{\circ}$. In addition, the gain of the improved antenna is still around $6.9 \mathrm{dBi}$, and the radiation patterns are stable, which ranges from 6.65 to $6.92 \mathrm{dBi}$ in $1.7-2.7 \mathrm{GHz}$.

\section{B. An Array of the Improved Dipole}

To satisfy the demand of high gain in mobile communication systems, a $1 \times 4$ antenna array is designed. As displayed in Fig. 9, the spacing between adjacent antenna elements is $120 \mathrm{~mm}$ to avoid grating lobe at high frequency. To maintain the wideband property, the antenna elements are fed by an equal-power splitter.

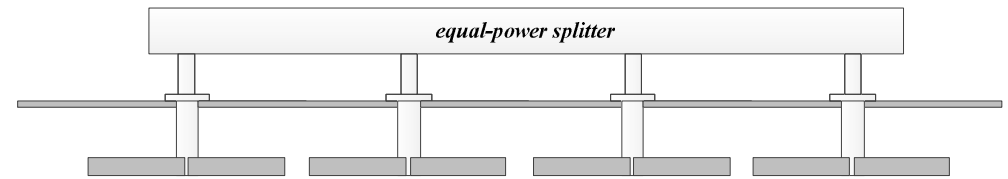

Fig. 9 Geometry of the $1 \times 4$ antenna array

The return loss of the array can fully cover the $2 \mathrm{G}, 3 \mathrm{G}$ and $4 \mathrm{G}$ band $(1.71 \mathrm{GHz}-2.69 \mathrm{GHz})$ as well. Here the pattern features are paid more attention. Fig.10-Fig.12 shows the simulated radiation patterns of the antenna array at $1.7 \mathrm{GHz}, 2.2 \mathrm{GHz}$ and $2.7 \mathrm{GHz}$.

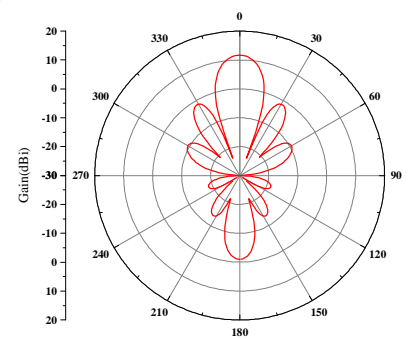

(a) E-plane

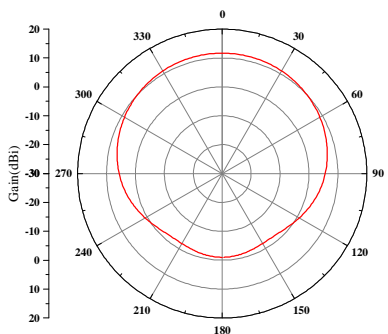

(b) H-plane

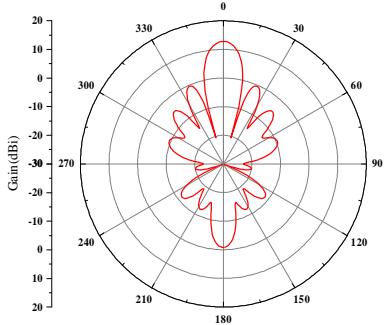

(a) E-plane

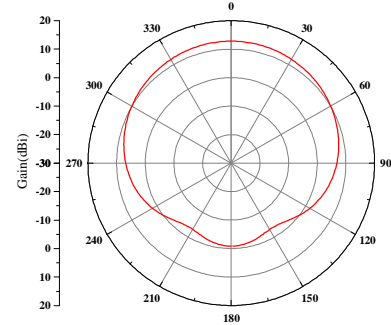

(b) H-plane

Fig. 10 Simulated radiation pattern at $1.7 \mathrm{GHz} \quad$ Fig. 11 Simulated radiation pattern at $2.2 \mathrm{GHz}$ 


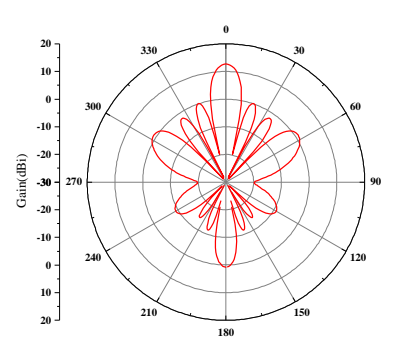

(a) E-plane

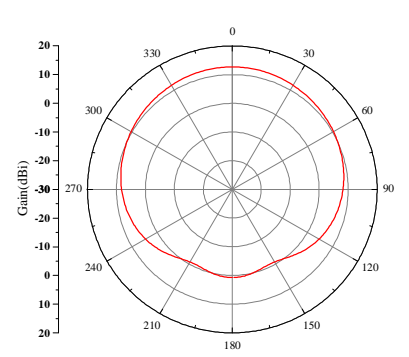

(b) H-plane

Fig. 12 Simulated radiation pattern at $2.7 \mathrm{GHz}$

The beamwidth of the H-plane keeps wide, which is still more than $120^{\circ}$. And the gain varies from $11.7 \mathrm{dBi}$ to $12.8 \mathrm{dBi}$ in the whole band. Consequently, the gain of the antenna is improved without affecting the impedance bandwidth and beamwidth in azimuth plane.

\section{Conclusions}

Base station antennas are expected to have broadband, high gain and wide beamwidth characteristics. However these three characteristics are contradictory. To cope with this problem, an improved dipole antenna is proposed. Compared with other work, the proposed antenna owns a broadband of 55.03\%, a wide beamwidth of no less than $120^{\circ}$ in azimuth plane, and a high gain of more than $6.65 \mathrm{dBi}$. Though the gain is not enough for the actual systems, it can be improved by arraying, and the simulated results of a $1 \times 4$ array have demonstrated it. With the benefits of the designed antenna, the mobile communication system can be simpler and the cost of the system will also be reduced, so this antenna is a good candidate for base station applications.

\section{References}

[1] Yejun He, Zhengzheng Pan, Xudong Cheng, and etc: A Novel Dual-Band, Dual-Polarized, Miniaturized and Low-Profile Base Station Antenna, IEEE Transactions on Antennas Wireless Propag. vol. 63,no. 12, pp. 5399-5408, December 2015.

[2] W. Sadowski and C. Peixeiro: Microstrip patch antenna for a GSM 1800 base station, in Proc. 12th Int. Conf. Microw. Radar, 1998, pp. 409-413.

[3] N. Herscovici and C. Christodoulou: Potentials of smart antennas in CDMA systems and uplink improvements, IEEE Antennas Propag. Mag., vol. 43, no. 5, pp. 172-177, Oct. 2001.

[4] K.M. Luk, and B. Wu: The magnetoelectric dipole-A wideband antenna for base stations in mobile communications, Proc. IEEE, vol. 100, no. 7, pp. 2297-2307, Jul. 2012.

[5] Luk, K.M., and Wu, B.: The magneto-electric dipole - a wideband antenna for base stations in mobile communications, Proc. IEEE, 2012, 100, (7), pp. 2297-2307.

[6] Yuehui C, Ronglin L, and Huanzhan F: A Broadband Dual-Polarized Planar Antenna for 2G/3G/LTE Base Stations, IEEE Antennas Wireless Propag. vol. 7, no. 9, pp. 4836-4840, 2014.

[7] Idayachandran G, and Nakkeeran R: 'Asymmetric folded dipole antenna with high front-to-back ratio for LTE base stations', IEEE Antennas Wireless Propag. vol. 15, pp. 869-872, 2016.

[8] Can Ding, Haihan S: Beamwidth control of base station antenna employing reflectors and directors, ISAP 2015.

[9] Shiwei Q, Jialin Li and Quan Xue: 'Bowtie Dipole Antenna with Wide Beamwidth for Base Station Applications’, IEEE Antennas Wireless Propag. Lett., vol. 7, pp. 293-295, 2007. 